EPJ Web of Conferences 64, 02008 (2014)

DOI: $10.1051 /$ epjconf/ 20146402008

(C) Owned by the authors, published by EDP Sciences, 2014

\title{
Long-term variability of low-mass X-ray binaries
}

\author{
E. Filippova ${ }^{1,2, a}$, M. Revnivtsev², and E. R. Parkin ${ }^{3}$ \\ ${ }^{1}$ ISDC, University of Geneva, Geneva, $\mathrm{CH}-1211$, Switzerland \\ ${ }^{2}$ Space Research Institute, Moscow, 117997, Russia \\ ${ }^{3}$ Research School of Astronomy and Astrophysics, ANU, Canberra, ACT 2611, Australia
}

\begin{abstract}
We consider modulations of mass captured by the compact object from the companion star's stellar wind in Low Mass X-ray Binaries with late type giants. Based on 3D simulations with two different hydrodynamic codes used Lagrangian and Eulerian approaches - the SPH code GADGET and the Eulerian code PLUTO, we conclude that a hydrodynamical interaction of the wind matter within a binary system even without eccentricity results in variability of the mass accretion rate with characteristic time-scales close to the orbital period. Observational appearances of this wind might be similar to that of an accretion disc corona/wind.
\end{abstract}

\section{Introduction}

It is generally accepted that in Low Mass X-ray Binaries (LMXB) the matter is accreted by a compact star via an accretion disc which is the dominant source of emission in the energy bands from optical to X-rays $[1,2]$. The currently favoured model for the X-ray flux variability of LMXBs is that of propagating fluctuations of mass accretion rate in the disc [3, 4]. The self-similar nature of such variations leads to the generation of flux variability with a power law type power density spectrum (PDS)[see e.g. 3]. The smallest frequency at which the viscosity fluctuations in the disc can generate a power law type flux variability, $f_{\text {visc }}$, corresponds to the viscous time-scale at the outer radius of the accretion disc. For typical parameters of the disk in LMXBs this frequency equals to $f_{\text {visc }} \sim(0.005-$ $-0.07) f_{\text {orb }}$, where $f_{\text {orb }}$ is an orbital frequency. At smaller frequencies there is a flattening of the flux variability PDS. An observational test of this model was presented by [5] where it was shown that LMXBs with long orbital periods $\left(P_{\text {orb }}>18 \mathrm{~h}\right)$ has a flattening of their PDSs at frequencies below $\sim 0.1 \times f_{\text {orb }}$, while LMXBs with smaller orbital periods $\left(P_{\text {orb }}<4\right.$ h) have PDSs in which the power law extends to much lower frequencies, and some (mostly "dippers", i.e. binaries, which due to high inclination demonstrate "dips" in their fluxes related to partial obscuration of the emitting regions at some orbital phases) have breaks around $\sim f_{\text {orb }}$. Systems in the work of [5] which have long orbital period also have giant/sub-giant optical companion. Systems with short orbital period have main sequence/white dwarf stars. In our work we emphasize that long orbital period binaries with giant/sub-giant companions have additional mass loss mechanism - stellar wind. The aim of the present work is to explore the temporal behaviour of accretion from the stellar wind of a late type giant in a long period LMXB and estimate the observational appearance of the stellar wind in such systems.

ae-mail: ekaterina.filippova@unige.ch 


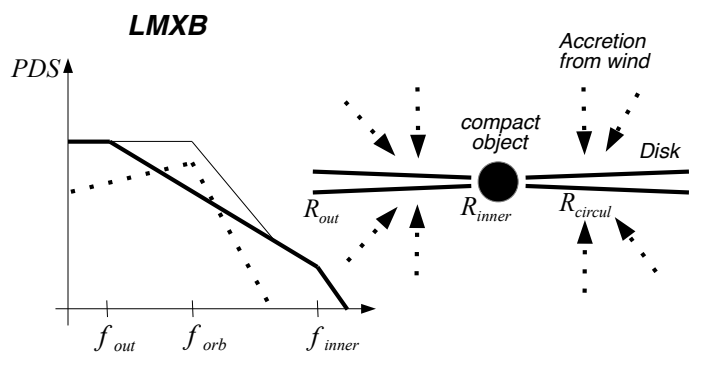

Figure 1. A schematic view of formation of the variability of LMXB's X-ray luminosity as a sum of mass accretion rate variability from a disc (thick line) and from the stellar wind of a giant companion (dotted line). The radii in the right panel have the following definitions: $R_{\text {out }}$ and $R_{\text {in }}$ are the outer and inner disc radii, respectively, and $R_{\text {circul }}$ is the circularization radius of the accreted wind matter. The resulted spectrum is shown by thin line on the plot.

\section{Model description}

A schematic view of accretion in an LMXB with a late type giant companion is shown in Fig. 1. We perform calculations for the brightest low mass X-ray binary, Sco X-1. We assume that optical star fills its Roche lobe and the velocity of the stellar wind corresponds to the star's escape velocity. For the Roche lobe radius of the optical star $R_{2, \mathrm{~L}}=8.5 \times 10^{10} \mathrm{~cm}$ we have $v_{\text {wind }} \sim 380 \mathrm{~km} \mathrm{~s}^{-1}$.

The mass loss rate from late type giants in compact binaries is not accurately known. Simple scaling laws for single stars predict a relatively low level of mass loss rate for giants of G-M type [6] but observations of late type giants in binaries indicate values up to $\sim 10^{-6} \mathrm{M}_{\odot} \mathrm{yr}^{-1}$ (e.g. GX 1+4 [7], and, CH Cyg - [8]). We estimated mass loss rate which is necessary to significantly influence the mass accretion rate onto the compact object which is $\sim(3-8) \times 10^{-8} \mathrm{M}_{\odot} \mathrm{yr}^{-1}$. For simulations we assume $\dot{M}_{\text {wind }}=5 \times 10^{-8}$ and $10^{-7} \mathrm{M}_{\odot} \mathrm{yr}^{-1}$.

In our simulations we do not take into account radiative cooling of the wind matter heated by shocks around the compact object. Our assumption is that cooling is compensated for by X-ray emission from the compact object via Compton heating.

\section{Results}

For the numerical simulations we use two codes which solve fluid equations in a different way: a SPH-code GADGET 2.7 [9] and the Eulerian grid-based code PLUTO [10].

Our simulations show that the accretion rate calculated as a mass flow through the "accretion radius" is not constant, but rather demonstrates fluctuations about a mean which appear as band limited noise even in the case of zero eccentricity binary system. The PDS of this variability in the simulations performed with GADGET is shown in Fig. 2 (solid line). It is clear that the accretion rate has an excess power at time-scales close to the orbital one (indicated by the vertical dashed line in the figure). Turning off all hydrodynamic forces, which is possible to do in the GADGET code (only gravitational and non-inertial forces then remain), we clearly see disappearance of the band limited noise, only the shot noise from the particles remains (see Fig. 2, dashed line). This shows that the mass accretion rate variability is caused by hydrodynamic interactions of matter in the binary.

The results of the PLUTO simulation also demonstrate a similar band limited noise in the mass accretion rate (see Fig. 3). Although the PDS shape from two codes is not exactly identical, their overall similarity is clear - both PDSs demonstrate excess of power at frequencies close to the orbital one. 

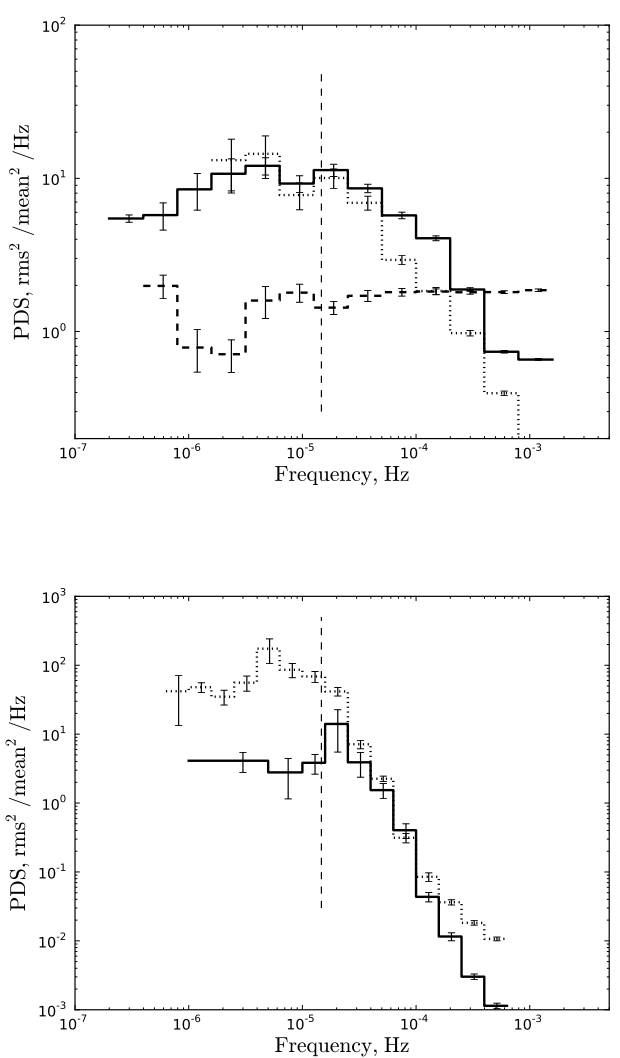

Figure 2. PDS for the mass accretion rate variability onto the compact object from the GADGET simulations. The solid histogram shows the PDS obtained in simulations with $1.2 \times 10^{5}$ particles, whereas the dotted histogram is for $6.6 \times 10^{5}$ particles. The dashed histogram shows the PDS for a simulation with hydrodynamic interactions turned off. The vertical line shows the orbital frequency of the binary system. The mass loss rate is $5 \times 10^{-8} \mathrm{M}_{\odot} \mathrm{yr}^{-1}$.

Figure 3. PDS for the mass accretion rate variability onto the compact object from the PLUTO simulations. The solid line shows the PDS for the case when the density in the accretion region is equal to the unperturbed density of the stellar wind at the distance of the compact object; the dotted line is for the case when the density is 3.5 times higher. The vertical line shows the orbital frequency of the binary system. The mass loss rate is $10^{-7} \mathrm{M}_{\odot} \mathrm{yr}^{-1}$.

\section{Observational appearance of the wind}

The stellar wind in a binary system might lead to absorption of X-rays emission due to the large column density of matter along the line of sight and the appearance of emission lines from the heated wind matter. Our simulations show that the absorbing column density in the binary along the line of sight can be $n_{H} L \sim(0.01-3) \times 10^{23} \mathrm{~cm}^{-2}$ (depending on the binary inclination, $\dot{\mathrm{M}}=10^{-7} \mathrm{M}_{\odot} \mathrm{yr}^{-1}$ ). However, the X-ray luminosity of the compact object can heat the wind matter to high temperatures, directly photo-ionize and significantly diminish the absorbing effect of it. Simple estimates of the ionization parameter $\xi=L_{\mathrm{x}} / n R^{2}$ (where $R$ is the distance of the matter with density $n$ from the X-ray source with luminosity $L_{\mathrm{x}}$ ) in the wind matter show that the distribution of emission measure of the matter over its ionization parameter has a broad peak around $\log \xi \sim 3.5$ which means that almost all elements will be completely ionized and absorbing disabled.

Using XSTAR code [11] we calculated spectrum of Sco X-1 X-ray emission when it has to pass through the wind matter. We assume that the matter forms a spherical cloud around the X-ray source. The temperature of the matter in the cloud is calculated iteratively until thermal equilibrium with the radiation is established. From Fig. 4 it is clear that the presence of the absorbing cloud results in formation the line forest in the X-ray spectrum which will be completely indistinguishable for X-ray instruments with poor energy resolution but might be detected by instruments with high resolution spectroscopy, for example, the X-ray microcalorimeters on Astro-H [12]. 


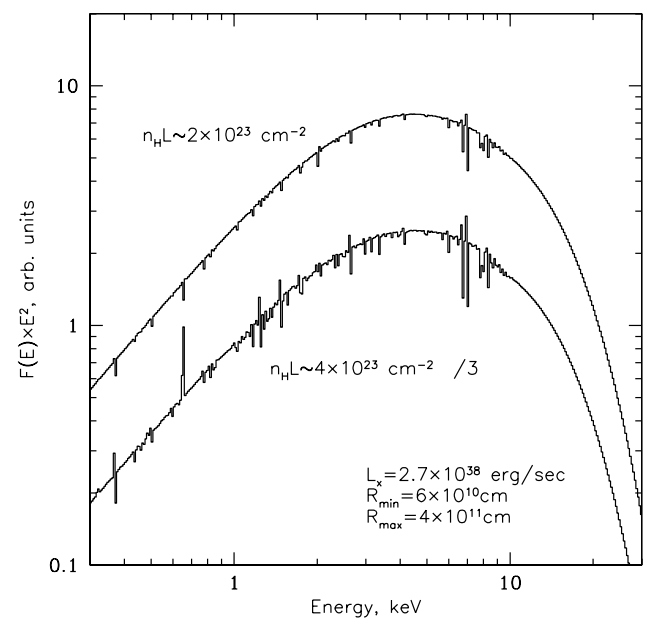

Figure 4. Spectra of emission of Sco X-1, seen through a uniform cloud of matter with inner radius $6 \times 10^{10} \mathrm{~cm}$, outer radius $4 \times 10^{11} \mathrm{~cm}$ and column density $2 \times 10^{23}$ $\mathrm{cm}^{-2}$ and $4 \times 10^{23} \mathrm{~cm}^{-2}$, calculated using the XSTAR code. The spectra are the sum of emission transmitted through the cloud and emission generated by the heated cloud itself.

\section{Conclusions}

With the help of 3D hydrodynamic simulations it is shown that the accretion rate from the stellar wind of a late type giant in an LMXB is intrinsically time variable with a characteristic time-scale close to the orbital period of the system even in the case of zero eccentricity orbit. The variability is caused by a hydrodynamical interaction of the stellar wind via the undeveloped turbulent motion (perturbed motion without significant vorticities) of the matter around compact object. The proposed mechanism for the generation of long time-scale variability (i.e. on the order of the orbital period) in the observed X-ray luminosity of persistent LMXBs with giant companions appears viable and should be explored further with more detailed numerical and observational studies. More detailed description of the work can be found in the paper [13].

\section{References}

[1] N. Shakura, R. Sunyaev, A\&A 24, 337 (1973)

[2] J. Frank, A. King, D. Raine, Accretion power in astrophysic (Cambridge university press, 2002)

[3] Y. Lyubarskii, MNRAS 292, 679 (1997)

[4] E. Churazov, M. Gilfanov, M. Revnivtsev, MNRAS 321, 759 (2001)

[5] M. Gilfanov, V. Arefiev, preprint (astro-ph/0501215) (2005)

[6] K. Schroder, M. Cuntz, ApJ 630, 73 (2005)

[7] D. Chakrabarty, M. van Kerkwijk, J. Larkin, ApJ 497, 39 (1998)

[8] M. Bogdanov, O. Taranova, Astron. Rep. 52, 403 (2008)

[9] V. Springel, MNRAS 364, 1105 (2005)

[10] A. Mignone, G. Bodo, S. Massaglia, T. Matsakos, O. Tesileanu, C. Zanni, A. Ferrari, ApJS 170, 228 (2007)

[11] T. Kallman, M. Bautista, ApJS 133, 221 (2001)

[12] K. Mitsuda, R. Kelley, K. Boyce, G. Brown, E. Costantini, M. DiPirro, Y. Ezoe, R. Fujimoto, J. Low Temperature Phys. 167, 795 (2012)

[13] E. Filippova, M. Revnivtsev, E.R. Parkin, MNRAS 1?? (2013) 